\title{
Surgical Technique and Considerations about Transanal Transection and Single-Stapled (TTSS) Anastomosis: The Search for a Perfect Anastomosis
}

\author{
Fábio Guilherme Campos ${ }^{1}$ Rafael Vaz Pandini ${ }^{1}$ (1) Leonardo Alfonso Bustamante-Lopez $^{1}$ \\ Sérgio Carlos Nahas ${ }^{1 \odot}$ \\ ${ }^{1}$ Colorectal Surgery Division, Gastroenterology Department, \\ Faculdade de Medicina, Universidade de São Paulo, São Paulo, Brazil
Address for correspondence Fábio Guilherme Campos, MD, Rua Padre João Manoel 222, Cj 120-São Paulo (SP), 01411-001, Brazil (e-mail: fgmcampos@terra.com.br). \\ J Coloproctol 2021;41(4):451-454.
}

\begin{abstract}
The evaluation of preventive measures and risk factors for anastomotic leakage has been a constant concern among colorectal surgeons. In this context, the description of a new way to perform a colorectal, coloanal or ileoanal anastomosis, known as transanal transection and single-stapled (TTSS) anastomosis, deserves an appreciation of its qualities, and a discussion about its properties and technical details. In the present paper, the authors review the most recent efforts aiming to reduce anastomotic dehiscence, and describe the

Keywords

- colorectal anastomosis

- rectal resection

- single-stapled

- transanal

- transection TTSS technique in a patient submitted to laparoscopic total proctocolectomy with ileal pouch-anal anastomosis for familial adenomatous polyposis. Surgical perception raises important advantages such as distal rectal transection under visualization, elimination of double-stapling lines (with cost-effectiveness and potential protection against suture dehiscence), elimination of dog ears, and the opportunity to be accomplished via a transanal approach after open, laparoscopic, or robotic colorectal resections. Future studies to confirm these supposed advantages are needed.
\end{abstract}

\section{Introduction}

One of the most feared complications after colorectal resections is the development of anastomotic dehiscence and its consequences, such as sepsis, reoperations, prolonged hospital stay, and the need for a stoma. ${ }^{1,2}$ Furthermore, leakage may affect the risk of recurrence, reduce survival, and delay adjuvant chemotherapy in oncological patients. ${ }^{3}$

The definition of anastomotic leakage is a controversial issue, and this complication has variable incidence rates. The risk factors are usually associated with location, clinical (age, clinical status, emergency setting, smoking, obesity, malnutrition, use of steroids, preoperative radiotherapy) and technical features (type of suture, surgeon experience). ${ }^{4}$ This complication has been documented in $8.1 \%, 6.4 \%$, and $11 \%$ of the cases after right colectomy, colonic cancer surgery, and rectal cancer surgery respectively ${ }^{5-7}$.

Thus, the performance of a safe anastomosis turns to be a major concern in colorectal surgery, the reason why the prevention of anastomotic leakage has been the topic of received

May 28, 2021

accepted after revision

August 6, 2021
DOI https://doi.org/

$10.1055 / \mathrm{s}-0041-1736295$.

ISSN 2237-9363.

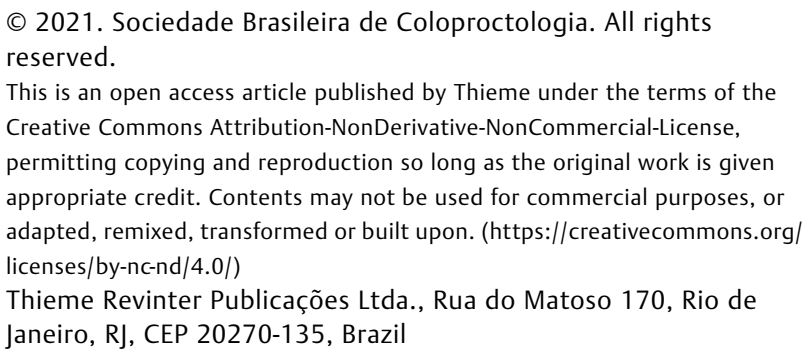


several investigations. Different strategies of administration of oral and intravenous antibiotics (with or without anterograde bowel cleansing) have gained great attention in the recent literature. On the other hand, the indication of intraabdominal drains or mechanical bowel preparation have raised lots of discussion. Especially for low rectal anastomosis, prevention and attenuation of pelvic sepsis with the use of fecal diversion is highly advisable and effective. ${ }^{8}$ More recently, the changing of resection margins using fluorescence angiography has been extensively evaluated to obtain a sound anastomosis. ${ }^{9}$

Issues related to the performance of anastomosis have also been a matter of concern. In this sense, the choice of number of suture planes, configuration (end-to-end, lateralto-end, latero-lateral), and suture type (hand-sewn versus mechanical) may have specific advantages in different clinical settings, although the rates of leakage do not differ significantly. For example, although the lateral-to-end anastomosis may provide better short-term function, an end-toend anastomosis may lead to less severe complications and reinterventions in the management of distal rectal tumors. ${ }^{10}$ The decision process concerning the type of anastomosis usually considers the surgeon's choice and experience.

It is widely recognized that the advent of mechanical staplers enabled the performance of safe low pelvic anastomosis, decreasing surgical length and enabling sphincter preservation. Performance of a low anterior resection is the main indication for the double-stapled technique (DST). The use of both linear and circular staplers brings the advantage of not requiring the placement of a pursestring suture deep on the distal rectum, a maneuver sometimes considered difficult when the pelvis is too narrow, in obese patients, or in the presence of large tumors. Moreover, the DST also leads to lower levels of local intraoperative contamination (as the rectal lumen is not exposed), and reduces the time and bleeding. ${ }^{11}$
However, the introduction of the linear stapler and its adaptation to transect the rectum may be eventually difficult, leading to oblique or multiple transverse firings (cartridges) necessary for rectal division. There is great evidence that the number of cartridges used is implicated in the occurrence of anastomotic dehiscence. ${ }^{12-16}$

Despite providing an easy and safe anastomosis, the DST creates critical points regarding the risk of disruption. One of them is the crossing of two stapling lines. More importantly, it creates lateral deformities at the extremes of the suture line, the so called "dog ears" 17 . These protrusions are regarded as a problem, as they are considered potential ischemic areas in the intersection of lateral stapling lines. ${ }^{14,18}$ For this reason, the invagination of this area and its inclusion in the circular stapler have been suggested. ${ }^{19}$ Whether or not this can reduce leaks remains to be established.

\section{TTSS: Technical Details}

Transanal transection and single-stapled anastomosis was first described by Spinelli et al., ${ }^{20}$ and it may be applied after total mesorectal excision with colorectal/coloanal anastomosis or after total proctocolectomy with ileal-pouch anal anastomosis (IPAA) for benign diseases. It takes the efficacy and reliability of total mesorectal excision (TME), and the combination of transanal transection (TT) with a singlestapled (SS) anastomosis from transanal TME (TaTME).

After the completion of the abdominal part of the dissection down to the pelvis, the perineal surgeon exposes the distal rectum with the aid of a Lone-Star retractor and/or a cylindrical anoscope, thus facilitating the recognition of the lesion and the demarcation of the distal margin.

Subsequently, a circular purse-string suture at the level chosen for rectal transection is performed (-Fig. 1, left to right). Once the rectum is closed, a circular full-thickness transection of the rectum wall is performed using electrocautery until it reaches the previous TME in the deep pelvis.

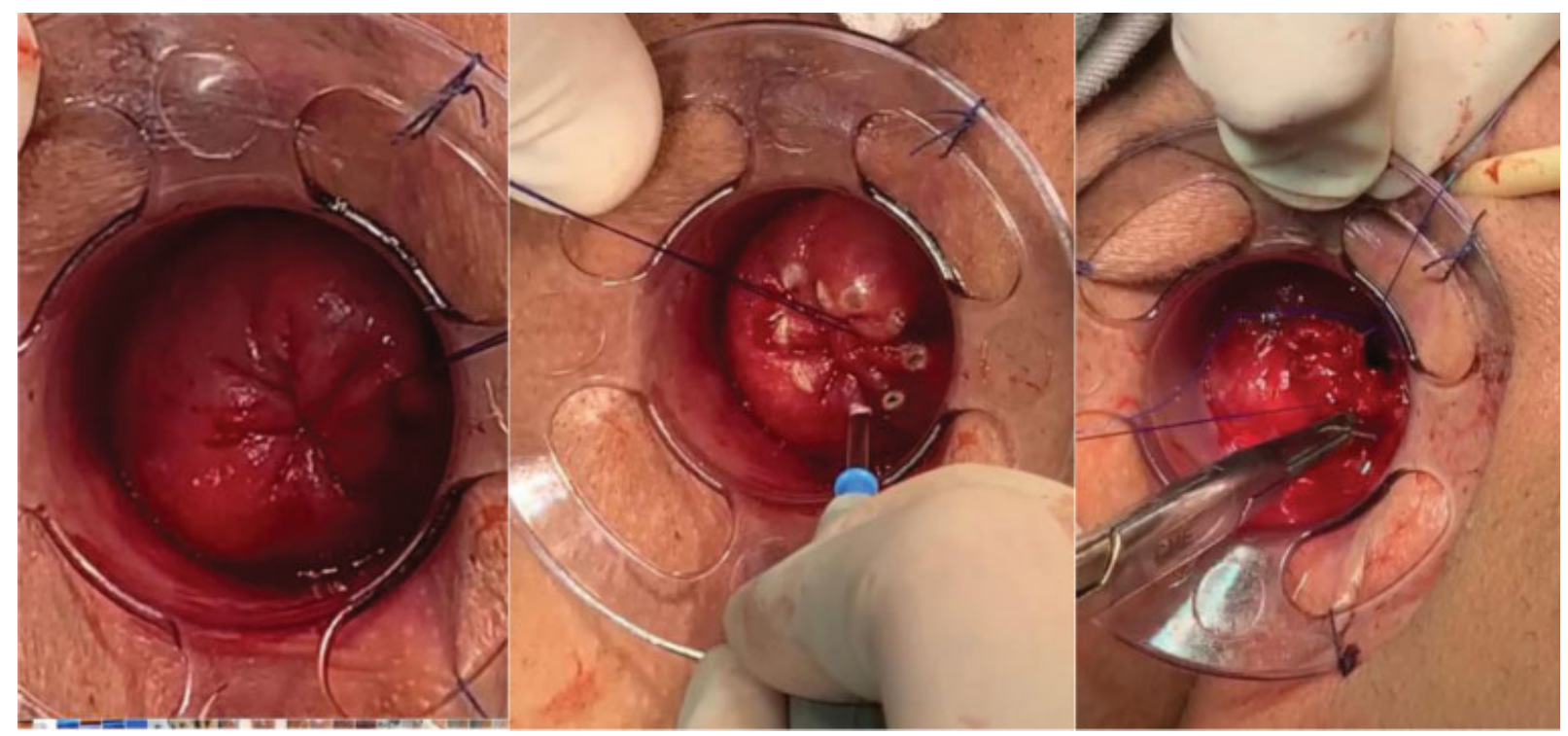

Fig. 1 Introduction of a rectal dilator and rectal suture (left), demarcation for the rectal section with electrocautery (middle), and a purse-string suture around the rectal cuff (right). 


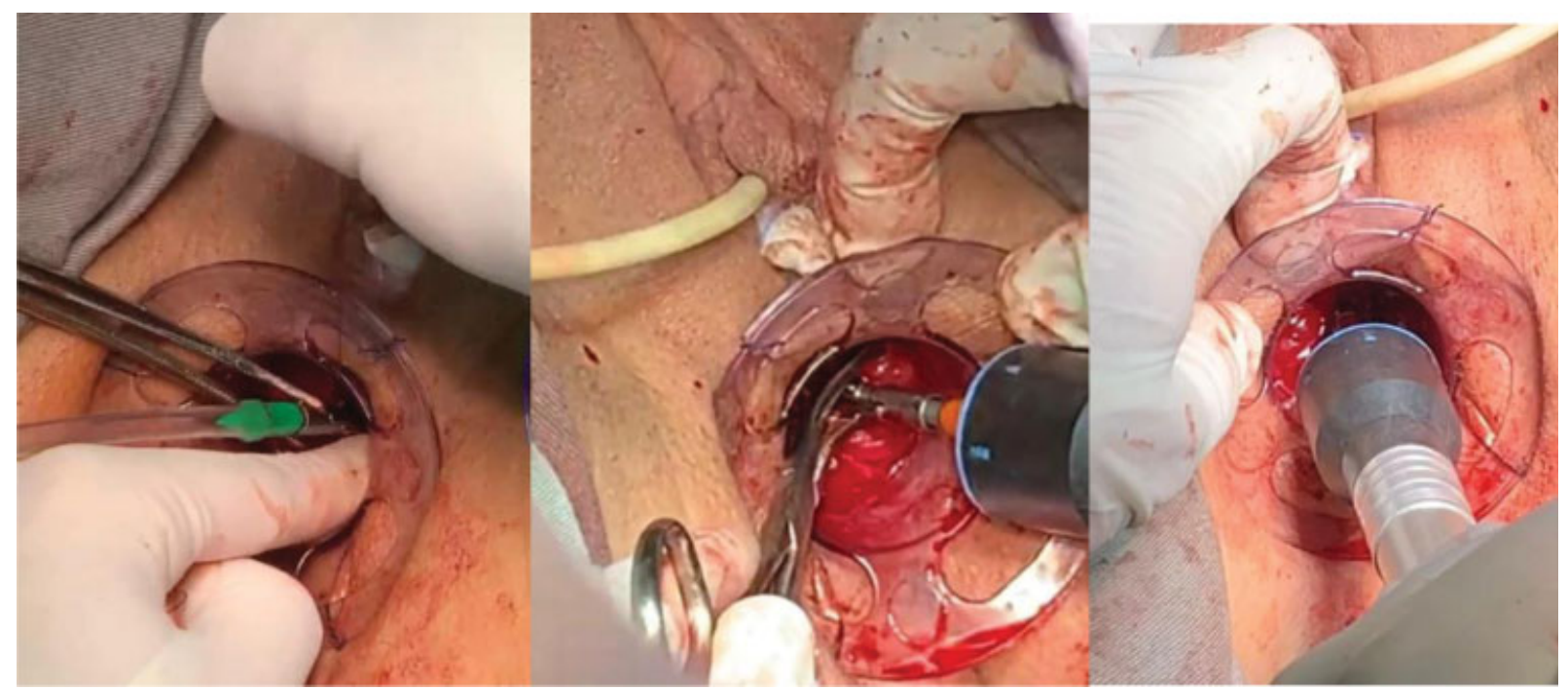

Fig. 2 (From left to right) Insertion and fixation of an anvil with a plastic tube passing through the opened rectal cuff (left), stapler coupling (middle), and single suture circular anastomosis (right).

After that, the colorectal specimen is pulled and retrieved through the perineum or through a Pfannenstiel incision (preferably with the presence of a wound protector). Transanal transection and single-stapled anastomosis enables the performance of rectal transection under visual control, avoiding multiple staple firings, which have been identified as risks factors for leakage. Another great advantage is the possibility to verify the distal margin and transect the rectum precisely. After that, a purse-string suture is made in the rectal cuff with a 2-0 prolene suture thread. After the definition of the proximal margin and its transection, the anvil of the circular stapler that is secured with a 2-0 suture (either in a lateral or terminal position) is placed, and a nasogastric tube is inserted in the tip of the anvil so the proximal colon can be externalized trough the anal canal and the purse-string suture of the rectal cuff can be tight. The circular stapler is inserted and connected to the anvil, enabling the performance of a single-stapled anastomosis followed by air-leak test or a rectal examination ( - Fig. 2, left to right). The SS anastomosis avoids cross-stapling and dog ears. Whether or not this can reduce leaks remains to be seen.

\section{Discussion}

Besides the continuous development in technology and the technical advances in rectal cancer surgery, performance of a safe anastomosis still presents many challenges regarding the distal dissection of the lower segment, the definition of the appropriate distal margin, and the avoidance of technical errors. The present report raises the main technical aspects involved in TTSS, a novel and interesting alternative to perform a low colorectal, coloanal, or ileoanal anastomoses.

This technique may be accomplished via a transanal approach after open, laparoscopic, or robotic colorectal resections. Development of the transanal approach aimed to surpass the limitations associated with minimally-invasive techniques to treat rectal cancer. ${ }^{21,22}$ Thus, it provides full visualization of the distal rectum, helping the surgeon to establish a correct distal resection margin and the precise transection level. ${ }^{22}$ The advantages of complete visual control have been recently recognized and emphasized during the learning phase of TaTME, raising the importance of performing a safe transection, especially in difficult cases (large tumors, obese patients, male pelvis).

Nowadays, patients with ulcerative colitis or familial adenomatous polyposis are preferably managed with double-stapled IPAA, as it is easier than the hand-sewn procedure, and provides better functional outcomes. However, there is a higher risk that residual mucosa may affect the postoperative symptoms and outcomes. ${ }^{23,24}$ In this context, these patients may also benefit from this technique, as it enables a more adequate evaluation of a potentially-diseased distal rectal mucosa. ${ }^{25}$

Moreover, TTSS is more cost-effective, as the use of another stapler to perform the transverse rectal transection (eventually requiring two or three firings) is dispensable. By avoiding the necessity of more than 2 transecting sutures, TTSS can also have a role in preventing leaks associated with this fact. Furthermore, its single-stapled configuration avoids the formation of dog ears.

Thus, if we consider that anastomotic dehiscence may occur due to stapler crossing or dog ears, TTSS turns out to be a promising strategy when colorectal, coloanal or ileoanal anastomoses are required. Simultaneously, it offers the key features of TaTME without the potential risks described during bottom-up dissections.

According to Spinelli, ${ }^{22}$ the main promising advantages of TTSS can be summarized as follows:

- It enables rectal transection under precise visual control;

- It may be performed after any surgical approach;

- Avoidance of tumor-cell dissemination;

- Cost-effectiveness in minimally-invasive surgery (as it eliminates transverse rectal transection); and

- It relies on established skills of the surgeon (the learning curve is not a problem). 
Conflict of Interests

The authors have no conflict of interests to declare.

\section{References}

1 Davis B, Rivadeneira DE. Complications of colorectal anastomoses: leaks, strictures, and bleeding. Surg Clin North Am 2013;93 (01):61-87

2 Kirchhoff P, Clavien PA, Hahnloser D. Complications in colorectal surgery: risk factors and preventive strategies. Patient Saf Surg 2010;4(01):5

3 Ha GW, Kim JH, Lee MR. Oncologic Impact of Anastomotic Leakage Following Colorectal Cancer Surgery: A Systematic Review and Meta-Analysis. Ann Surg Oncol 2017;24(11):3289-3299[PMID: 28608118 DOI: 10.1245/s10434-017-5881-8]

4 Bruce J, Krukowski ZH, Al-Khairy G, Russell EM, Park KG. Systematic review of the definition and measurement of anastomotic leak after gastrointestinal surgery. Br J Surg 2001;88(09): 1157-1168

5 The 2015 European Society of Coloproctology collaborating group. The relationship between method of anastomosis and anastomotic failure after right hemicolectomy and ileo-caecal resection: An international snapshot audit. Colorectal Dis 2017; 19:e296-e311

6 Krarup PM, Jorgensen LN, Andreasen AH, Harling HDanish Colorectal Cancer Group. A nationwide study on anastomotic leakage after colonic cancer surgery. Colorectal Dis 2012;14(10): e661-e667

7 Paun BC, Cassie S, MacLean AR, Dixon E, Buie WD. Postoperative complications following surgery for rectal cancer. Ann Surg 2010; 251(05):807-818

8 Caputo D, Angeletti S, Fiore M, et al. Delayed surgery after radiochemotherapy for rectal adenocarcinoma is protective for anastomotic dehiscence: a single-center observational retrospective cohort study. Updates Surg 2020;72(02):469-475

9 Kiran RP, Murray AC, Chiuzan C, Estrada D, Forde K. Combined preoperative mechanical bowel preparation with oral antibiotics significantly reduces surgical site infection, anastomotic leak, and ileus after colorectal surgery. Ann Surg 2015;262(03):416-425, discussion 423-425

10 Planellas P, Farrés R, Cornejo L, et al. Randomized clinical trial comparing side to end vs end to end techniques for colorectal anastomosis. Int J Surg 2020;83:220-229. Doi: 10.1016/j. ijsu.2020.09.039, Online ahead of print.

11 Knight CD, Griffen FD. An improved technique for low anterior resection of the rectum using the EEA stapler. Surgery 1980;88 (05):710-714

12 Kim HJ, Choi GS, Park JS, Park SY. Comparison of intracorporeal single-stapled and double-stapled anastomosis in laparoscopic low anterior resection for rectal cancer: a case-control study. Int J
Colorectal Dis 2013;28(01):149-156[PMID: 23014975 DOI: 10.1007/s00384-012-1582-8]

13 Park JS, Choi GS, Kim SH, et al. Multicenter analysis of risk factors for anastomotic leakage after laparoscopic rectal cancer excision: the Korean laparoscopic colorectal surgery study group. Ann Surg 2013;257(04):665-671

14 Roumen RM, Rahusen FT, Wijnen MH, Croiset van Uchelen FA. "Dog ear" formation after double-stapled low anterior resection as a risk factor for anastomotic disruption. Dis Colon Rectum 2000;43(04):522-525

15 Kawada K, Hasegawa S, Hida K, et al. Risk factors for anastomotic leakage after laparoscopic low anterior resection with DST anastomosis. Surg Endosc 2014;28(10):2988-2995

16 Braunschmid T, Hartig N, Baumann L, Dauser B, Herbst F. Influence of multiple stapler firings used for rectal division on colorectal anastomotic leak rate. Surg Endosc 2017; 31(12):5318-5326[PMID: 28634627 DOI: 10.1007/s00464-0175611-0]

17 Zhuo C, Liang L, Ying M, et al. Laparoscopic Low Anterior Resection and Eversion Technique Combined With a Nondog Ear Anastomosis for Mid- and Distal Rectal Neoplasms: A Preliminary and Feasibility Study. Medicine (Baltimore) 2015;94(50):e2285

18 Rudi M, Roumen H. "Dog ear" formation after double stapled low anteriorresection as a risk factor for anastomotic disruption. Dis Colon Rectum 2000;43:4

19 Torres VF, Decanini TC, Belmonte MC, et al. Aparatos de suturamecánica. Historia, desarrollo y experiencia en el Hospital Central Militar. Rev Gastroenterol Mex 1992;57:132-135

20 Spinelli A, Carvello M, D'Hoore A, Foppa C. Integration of transanal techniques for precise rectal transection and single-stapled anastomosis: a proof of concept study. Colorectal Dis 2019;21(07): 841-846

21 Penna M, Hompes R, Arnold S, et al; TaTME Registry Collaborative. Transanal total mesorectal exci- sion: international registry results of the first 720 cases. Ann Surg 2017;266(01):111-117

22 Spinelli A. The rationale for expanding transanal transection and single-stapled anastomosis from transanal total mesorectal excision to open, laparoscopic and robotic resections. Colorectal Dis 2019;21(09):991

23 Ganschow P, Treiber I, Hinz U, Leowardi C, Büchler MW, Kadmon M. Residual rectal mucosa after stapled vs. handsewn ileal Jpouch-anal anastomosis in patients with familial adenomatous polyposis coli (FAP)-a critical issue. Langenbecks Arch Surg 2015; 400(02):213-219

24 Campos FG, Imperiale AR, Seid VE, et al. Rectal and pouch recurrences after surgical treatment for familial adenomatous polyposis. J Gastrointest Surg 2009;13(01):129-136

25 de Lacy FB, Keller DS, Martin-Perez B, et al. The current state of the transanal approach to the ileal pouch-anal anastomosis. Surg Endosc 2019;33(05):1368-1375 